\title{
Comparison of Vertical and Inclined Toothbrush Filaments: Impact on Shear Force and Penetration Depth
}

\author{
Frank Goldschmidtboeing1,* - Alexander Doll ${ }^{2}$ - Ulrich Stoerkel ${ }^{2}$ - Sebastian Neiss ${ }^{1}$ - Peter Woias ${ }^{1}$ \\ 1 University of Freiburg, Department for Microsystems Technology, Germany \\ 2 Procter \& Gamble Manufacturing GmbH, Oral-B Laboratories, Germany
}

This paper presents a semi-analytical model to describe the action of vertical and $16^{\circ}$-inclined toothbrush filaments onto flat tooth surfaces and into approximal areas. The theory is based on the analytical solution of the beam equation about normal and transversal (shear) forces that deform the filaments. A 15\% increase of penetration depth (approximal area model) and a 60\% increase of transversal force (tooth surface model) for an inclination angle of $16^{\circ}$ compared to a vertical filament were determined. Furthermore, that $16^{\circ}$-inclined filaments are able to transfer higher shear forces to the tooth compared to vertical ones was shown experimentally, which could account for the higher efficiency of plaque removal by inclined filaments compared to vertical filaments. The theoretical findings for manual and oscillating-rotating toothbrushes are discussed, and implications for their design are drawn.

Keywords: vertical and inclined toothbrush filaments, shear forces, penetration depth, power toothbrush, mechanical plaque removal, oscillating-rotating technology

\section{INTRODUCTION}

Dental plaque is an oral biofilm that adheres to the teeth and consists of many species of bacterial cells, salivary polymers and microbial extracellular products.

Without proper control, dental plaque is known to be a causative and perpetuating factor of several common oral diseases, including gingivitis, periodontitis and caries. Therefore, the removal of plaque biofilm is the primary goal of oral hygiene.

Dental plaque formation occurs in several steps [1], starting with a layer of adsorbed molecules of bacterial and salivary origin. Subsequently, initial colonizing bacteria (generally streptococci) attach to this layer. Secondary colonizers attach via coadhesion and make the biofilm more diverse, resulting in a multispecies community. A biofilm matrix is formed by the attached bacteria via the synthesis of extracellular polymers. The combined metabolic activity of different bacteria can break down complex host macromolecules, which subsequently leads to the development of food chains. As a result, oral biofilms are highly organized, both structurally and functionally.

While numerous products are commonly recommended to achieve optimal plaque levels, including dental floss, fluoride dentifrice, and rinse, the toothbrush is a core component of any oral hygiene regimen. The main purpose of brushing teeth remains the effective disruption and removal of dental plaque. The plaque biofilm grows on teeth, resulting in a complex structure, depending on the composition, age and location, with different mechanical properties.
Independent of the particular structure and mechanical strength of the biofilm, it is common knowledge that mechanical energy and in particular shear forces are needed to remove the adhering plaque from the tooth surface [2]. Also well accepted is the fact that the tips of toothbrush filaments create higher shear forces than the respective flanks.

For effective plaque removal with the desired result of clean and healthy teeth and gums, several factors are important. To start with, the filament tips have to reach the plaque, which is easy to achieve for a large flat surface, but difficult for the approximal area around the connection sites of neighbouring teeth. Although access to those hard-to-reach areas is essential, access alone is not sufficient since the applied forces have to be greater than the adhesion and cohesion forces of the biofilm to destroy and remove it from the tooth surface.

Toothbrush innovations have to simultaneously address several consumer needs, with the improvement of the efficacy of plaque removal during the brushing routine of users being an important aspect for both manual- and power-driven toothbrushes. In 1999, Oral-B introduced an innovative manual toothbrush, called CrossAction ${ }^{\circledR}$. Detailed investigation into the action of filaments during brushing was part of the development. As a result of the development process, the filament tufts were arranged in a unique CrissCross $^{\circledR}$ design with filaments inclined at $16^{\circ}$ (Fig. 1). Since its creation, the brush has proven to be a leading manual toothbrush that maximizes plaque removal, regardless of how the user brushes, which is supported by laboratory investigations, as well as clinical studies. Laboratory studies [3] have 
demonstrated that this development significantly enhances approximal area penetration and cleaning effectiveness in comparison to an identical brush head with parallel, vertical (rather than inclined) filaments. Comparisons with more than 80 leading manual toothbrushes from around the world have demonstrated a consistent, significant advantage for the CrossAction ${ }^{\circledR}$ toothbrush in approximal area penetration and cleaning effectiveness [3]. According to a five-year literature review (2000 to 2005) [4] of clinical research, 14 single-brushing clinical comparisons and two longer-term clinical studies have demonstrated consistently greater plaque removal for the Oral-B CrossAction brush over the equivalent commercial standards, specifically for effective approximal cleaning. Several additional studies since then have confirmed those results [5] to [7]. Longerterm research also demonstrates significant benefits for CrossAction ${ }^{\circledR}$ in controlling gingivitis [8]. As with all Oral-B brushes and brush heads, CrossAction filaments are end-rounded to ensure gentle cleaning.

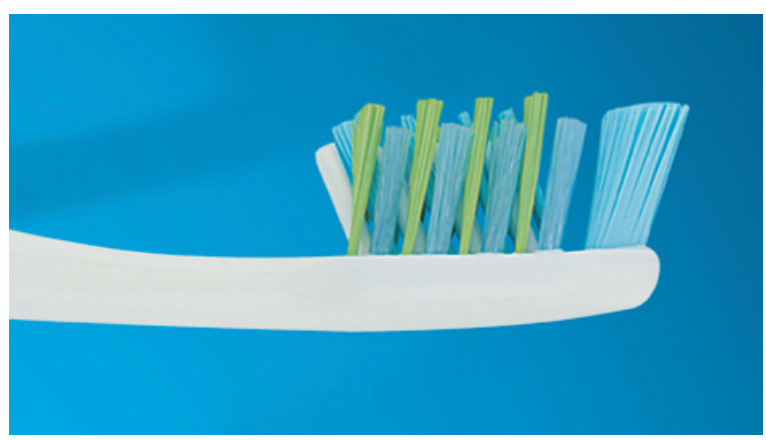

Fig. 1. Oral-B CrossAction ${ }^{\circledR}$ manual toothbrush

Power-driven toothbrushes have additional features for supporting the shear forces created by the filaments, and these features have been shown to enhance plaque removal performance even further and lead to increased performance in comparison to manual toothbrushes [9] and [10]. An example of such a feature is the $3 \mathrm{D}$ pulsation of the Oral-B Professional Care oscillating-rotating product line, which supports filament penetration into plaque. The pulsation provides an applied force in the direction along the filament axis, which leads to so-called poking events that provide additional shear forces (typically 340 pulsations per second). Other important features of power driven brushes are the short stroke length and the significantly higher frequency of motion (typically 78 oscillations per second) compared to manual brushes (typically 2 to 3 strokes per second). The frequent changes of direction consequently lead to a higher number of poking events. Shear forces are most effective at turning points of direction; thus, the combination of short movements with a high number of turning points at high frequency synergistically optimizes plaque removal efficacy. However, it is important that the stroke length remains long enough to allow the filaments to change their orientation and to apply sufficient shear forces.

In order to investigate and analyse the effect of combining the current advantages of power toothbrushes (3D motion of pulsations combined with oscillation/rotation) with the proven features of the CrossAction ${ }^{\circledR}$ manual toothbrush (i.e. the arrangement of most tufts at an inclined $16^{\circ}$ angle), in-vitro experiments to explore this technology over current tuft geometries were designed. These in-vitro experiments may help to predict beneficial in-use relevance and thus demonstrate potential advances in brush design.

This paper is structured as follows: at the start, a first-principle model to describe the filament shapes and the forces onto the tooth surface by a semianalytical approach is presented. Thereafter, the resulting equations are solved, and the filament shapes are visualized in the solution section. After that, the results of a numerical simulation to the problem to judge the applicability of the simplified analytical model from the first section are presented. Then, an experimental validation of the hypothesis that $16^{\circ}$-inclined filaments transfer higher shear forces to the tooth in comparison to vertical filaments is presented. In the discussion section, some conclusions for optimizing toothbrush design, especially relevant for the design of oscillating-rotating power brushes, are drawn.

\section{FIRST-PRINCIPLE MODELLING}

The aim here is to develop and analyse a simplified theoretical model to understand the basic effects of the inclination angle of toothbrush filaments on the shear force and on the penetration depth into approximal areas. Two types of toothbrushes are considered: a manual brush (Fig. 1) and the Oral-B oscillatingrotating power brush (Fig. 2), both with vertical and $16^{\circ}$-inclined end-rounded filaments.

For the sake of simplicity, both types of filament motion, the linear motion of a manual brush (Fig. 1) and the oscillating-rotating motion of a power brush (Fig. 2), are mapped onto a linear movement along the $x$ axis.

The stroke length of a standard manual brush is assumed to be typically $10 \mathrm{~mm}$ while the typical 
stroke lengths of oscillating-rotating brush heads are in the range of $3.0 \mathrm{~mm}$ (inner ring) and $5.1 \mathrm{~mm}$ (outer ring) for the free moving head, respectively, and $2.3 \mathrm{~mm}$ (inner ring) and $4.0 \mathrm{~mm}$ (outer ring) for the oscillating-rotating brush head with a total recommended maximum brushing force of $2 \mathrm{~N}$.

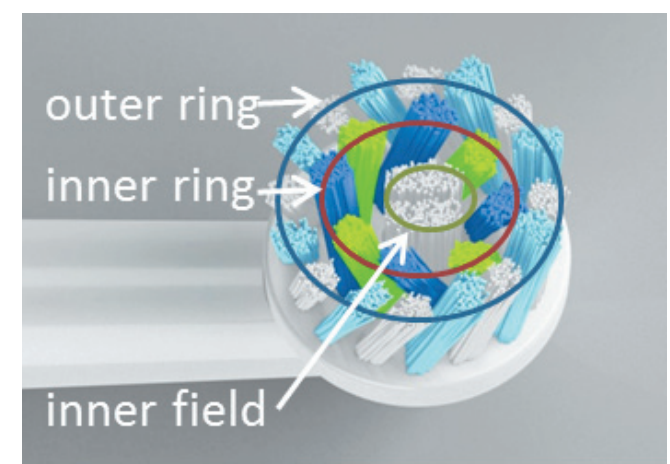

Fig. 2. Sketch of the oscillating-rotating brush with vertical and $16^{\circ}$-inclined filaments

The mapping of the oscillating-rotating motion to a linear one obviously introduces some error, as no radial forces and displacements are considered, but as these effects are understood to be small, a qualitative deviation from the real behaviour is not expected. Two geometric models are discussed (Fig. 3).

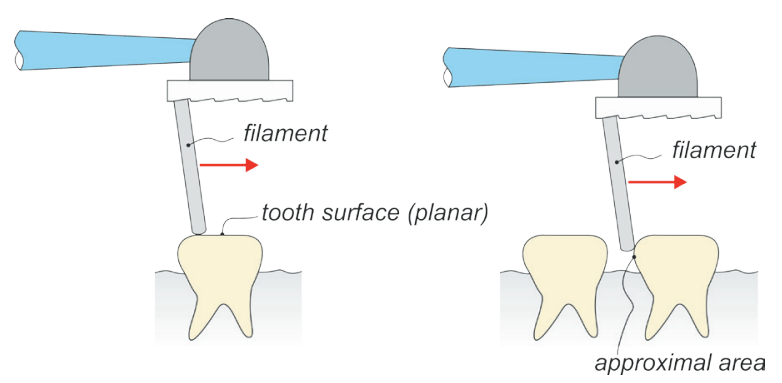

Fig. 3. Simplified tooth surface model (left) and approximal area model (right)

The first model describes the tooth surface as a perfect plane and this simplified tooth surface model is used to calculate the occurring forces. The second model describes the penetration depth of the filament into an approximal area. Both models were developed to allow for the analytical solution of the filament deformations, to show the principle effects of inclined filaments. More detailed tooth models could, for example, be generated by the digitization methods described by Budak et al. [11].

Nevertheless, the focus is on the demonstration of very basic effects that are meaningful for any tooth geometry and do not depend on details of the tooth surface. Therefore, the simplest possible tooth model was chosen.

\subsection{The Tooth Surface Model}

The model is schematically visualized in Fig. 4. The sketch is rotated so that the filament can be treated as a cantilever in the $x$-direction with the $z$-direction facing downwards. The length of the filament is symbolized by $L$ and the distance between the brush, and the perfectly planar tooth surface is given by $d$. The inclination angle $\alpha$ is defined as the angle between the perpendicular axis of the brush and the non-deformed filament. The position of the filament tip on the tooth surface is given by $w_{t i p}$ with $w_{t i p}=0$ at the incidence of the non-deformed filament. The filament's projection onto the x-axis is denoted as $L_{x}$. The normal force $F_{x}$ (defined in negative $x$-direction) and the transverse force $F_{z}$ (defined in negative $x$-direction) deform the filament. Their projections in normal and tangential directions to the tooth surfaces are called the contact force $F_{n}$ and shear force $F_{t}$, respectively.

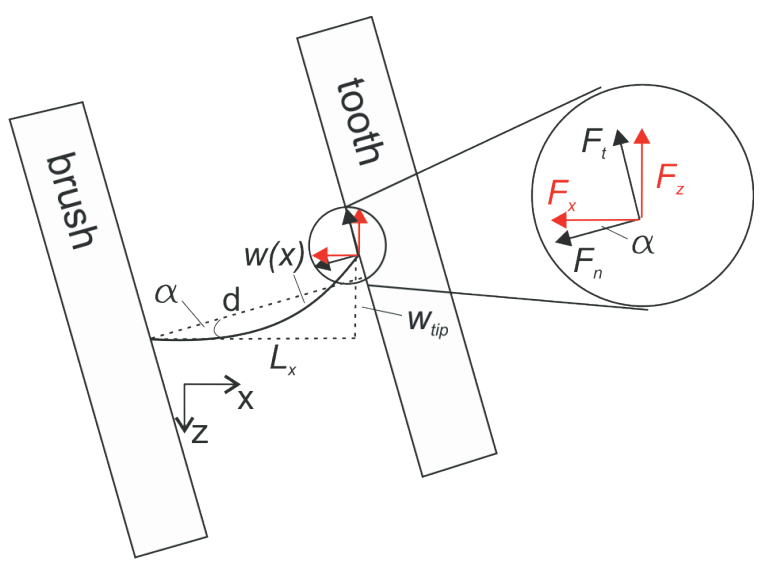

Fig. 4. Idealized 2D-model of the Filament

The deformation of the filament $w(x)$ is defined by the differential beam equation (Eq. (1)) [12], where $E$ denotes the Young's modulus of the filaments and $I$ its second moment of area. Strictly speaking, this approach is only valid for small displacements and the filaments might show considerably high displacements. Therefore, its accuracy is checked in the numerical simulations section.

$$
w^{(4)}(x)+\lambda^{2} \cdot w^{\prime \prime}(x)=0, \quad \lambda^{2}=\frac{F_{x}}{E I} .
$$

This differential equation must be solved with the four boundary conditions given in Eq. (2) for the bearing (brush side) and for the tip (tooth side). 


$$
\begin{gathered}
w(0)=0, \quad w^{\prime}(0)=0, \quad w\left(L_{x}\right)=w_{\text {tip }}, \\
M\left(L_{x}\right)=-E I \cdot w^{\prime \prime}(x)=0 .
\end{gathered}
$$

The last part of Eq. (2) states that the tooth does not imply any torque on the filament. The solution of Eq. (1) with boundary conditions of Eq. (2) is given by Eq. (3).

$$
\begin{gathered}
w(x)=\frac{w_{t i p}}{\tan \left(\lambda \cdot L_{x}\right)-\lambda \cdot L_{x}} \cdot\{\sin (\lambda \cdot x)- \\
\left.-\lambda \cdot x+\tan \left(\lambda \cdot L_{x}\right) \cdot(1-\cos (\lambda \cdot x))\right\} .
\end{gathered}
$$

The only remaining unknown is the axial force parameter $\lambda$, as the projection length $L_{x}$ and the tip displacement wtip are related by the geometrical condition Eq. (4). The axial force parameter $\lambda$ is determined from the fact that the curved filament length equals the straight filament length $L$, Eq. (5).

$$
\begin{gathered}
L_{x}=\frac{d}{\cos (\alpha)}+w_{t i p} \cdot \tan (\alpha), \\
L=\int_{0}^{L_{x}} \sqrt{1+w^{\prime}(x)^{2}} d x .
\end{gathered}
$$

Eq. (5) with the bending line from Eq. (3) and the projected length $L_{x}$ from Eq. (4) can be solved numerically for the parameter $\lambda$ in dependence on $\alpha, d, L$ and $w_{t i p}$. This numerical solution yields an infinite number of positive solutions for the axial force parameter $\lambda$. The lowest value for $\lambda$ is chosen because it is the only value that produces physically stable solutions. With this solution, the forces $F_{x}$ and $F_{z}$ can be calculated directly according to Eq. 6, while the projections $F_{n}$ and $F_{t}$ are calculated from Eq. (7).

$$
\begin{gathered}
F_{x}=E I \cdot \lambda^{2}, \quad F_{z}=E I \cdot\left(\lambda^{2} \cdot w^{\prime}\left(L_{x}\right)+w^{\prime \prime \prime}\left(L_{x}\right)\right), \\
F_{t}=F_{z} \cdot \cos (\alpha)+F_{x} \cdot \sin (\alpha), \\
F_{n}=-F_{z} \cdot \sin (\alpha)+F_{x} \cdot \cos (\alpha) .
\end{gathered}
$$

The limiting values for $w_{t i p}$ are given by the solutions for $\lambda=0$, i.e. solutions with zero axial force. These solutions are found by taking the limit $\lambda \rightarrow 0$ of Eq. (3), which is given in Eq. (8). This solution is obviously the pure bending solution that could be found directly by integrating the beam Eq. (1) with zero axial force.

$$
w(x)=\frac{w_{t i p}}{L_{x}^{3}} \cdot\left(\frac{3}{2} \cdot L_{x} \cdot x^{2}-\frac{1}{2} \cdot x^{3}\right) .
$$

The resulting tip displacement can then be calculated from the length condition Eq. (4), which yields two solutions $w_{\min }<0$ and $w_{\max }>0$

To judge whether the calculated bending lines $w(x)$ represent stable states, a stability analysis for the steady state has to be performed. Stability is obtained as long as the second derivative of the elastically stored energy $W$ with the tip displacement $w_{\text {tip }}$ is positive. This requirement is equivalent to a positive slope of the transversal force $F_{t}$ versus the tip displacement $w_{\text {tip }}$ (Eq. 9). This condition holds because the normal force $F_{n}$ does not perform any work on the beam.

$$
\frac{\partial^{2} W}{\partial w_{t i p}^{2}}>0 \Leftrightarrow \frac{\partial F_{t}}{\partial w_{t i p}}>0 .
$$

\subsection{Model for Penetration into Approximal Areas}

The model for an approximal area is shown in Fig. 5. The rounded edge of the tooth is modelled with a radius of $R=2 \mathrm{~mm}$. The relative distance between the bearing of the filament and the beginning of the rounded shape is called $z_{0}$. The position of the tip on the rounded shape is given by the angle $\phi$. The modelling concept is the same as for the tooth surface model, but now two variables have to be considered. While for the tooth surface model all forces merely depend on the tip displacement $w_{\text {tip }}$, here the position $z_{0}$ and the angle $\phi$ have to be considered. As we have only changed the geometry compared to the prior model, only the geometrical Eq. (3) and the Eq. (7) have to be replaced. Eq. (3) is replaced by Eq. (10) that relates the modelling variables $L_{x}$ and $w_{t i p}$ to the filament position $z_{0}$ and the tip position $\phi$.

Eq. (10) can be derived from Fig. 5 given that the opposite edges of the large rectangle are equal.

$$
\begin{gathered}
L_{x}=(d+R) \cdot \cos (\alpha)- \\
-R \cdot \cos (\alpha+\phi)-z_{0} \cdot \sin (\alpha), \\
w_{t i p}=-z_{0} \cdot \cos (\alpha)- \\
-(d+R) \cdot \sin (\alpha)+R \cdot \sin (\alpha+\phi) .
\end{gathered}
$$

Furthermore, the equations for the normal force $F_{N}$ and the tangential force $F_{T}$ have to be replaced by Eq. (11).

$$
\begin{aligned}
& F_{N}=F_{z} \cos (\alpha+\phi)+F_{x} \cdot \sin (\alpha+\phi), \\
& F_{T}=F_{z} \cdot \sin (\alpha+\phi)-F_{x} \cdot \cos (\alpha+\phi) .
\end{aligned}
$$




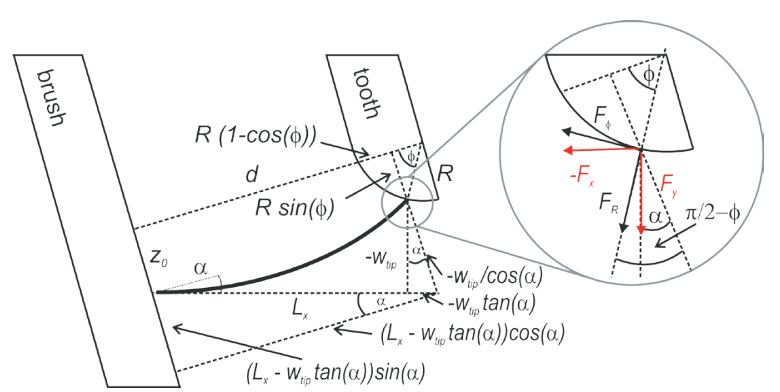

Fig. 5. Idealized 2D-model of the filament in an approximal area

\section{SOLUTION}

\subsection{Tooth Surface Model and Brush Geometry}

Filaments with seven different angles $\alpha$, from 0 to $24^{\circ}$, are compared. The lengths of the filaments $L$ are adjusted in such a way that the thickness of the non-deformed brush $d_{0}$ is constant $\left(L=d_{0} / \cos (\alpha)\right)$. The round-shaped filaments have a diameter of 6 mil $(0.152 \mathrm{~mm})$ and a Young's modulus of $E=2.7$ $\mathrm{GPa}$ and, therefore, a resulting bending stiffness of $E I=7.1510^{8} \mathrm{Nm}^{2}\left(I=\pi / 64 \cdot d^{4}\right)$.

\subsubsection{Solution: Bending Lines}

Each brush geometry is examined for four different constant distances $d$ between brush and tooth: $d=7.46,7.71,7.96$ and $8.21 \mathrm{~mm}$, which means that the filaments are deformed in such a way that the distance between the brush head and tooth is reduced by $1,0.75,0.5$ and $0.25 \mathrm{~mm}$, respectively, compared to the no-load condition with a distance of $d_{0}=8.46 \mathrm{~mm}$. The deformation of $1 \mathrm{~mm}$ corresponds roughly to the recommend maximum normal force of the Oral-B Professional Care product line. First, the limiting bending lines with zero axial force are determined from Eq. (8) with Eq. (5). Then, the bending lines for 100 equidistant tip displacements $w_{\text {tip }}$ between $w_{\text {min }}$ and $w_{\max }$ are calculated from Eq. (3) with the length condition, Eq. (5). The forces $\left(F_{x}, F_{z}, F_{n}, F_{t}\right)$ are then easily determined from Eq. (6) and (7).

The filament movement is described via Figs. 6 and 7. It must be noted that the model is based on static forces and displacements, while the real process is inherently dynamic; nevertheless, some important issues may be derived from the static curves. The model yields two types of solutions: the "sticking" solution and the "sliding/sticking" solution. The sticking solutions (i.e. the solution violating the stability criterion in Eq. (9)) refer to the filament shapes that occur when the filament tip is stuck to the tooth surface, while the sliding/sticking solutions describe shapes when the filament tip slides along the tooth surface with constant filament shape, and the friction force equals the bending force of the filament. If the friction force exceeds the bending force, the tip again becomes stuck to the surface, and the filament shape evolves from sliding/sticking to a pure sticking solution. The friction force typically varies during a brushing event due to accumulated plaque or toothpaste particles. Therefore, a sliding filament typically becomes stuck after a while.

In Fig. 6, the bending lines for a simplified toothbrush filament are shown. Here $x^{\prime}$ is the coordinate along the tooth surface, while $z^{\prime}$ is the coordinate normal to the tooth surface. These coordinates are used to visualize the results in a clear manner. The filament shapes from the original $x, z$ coordinate system are rotated to the new $x^{\prime}, z^{\prime}$ system. Different brush head positions are plotted to clarify the filament behaviour on a simplified surface when moving the brush head forward (from left to right in the diagrams as in Fig. 3). Fig. 6a shows the bending line just after touching the surface. With further movement, the tangential force increases. In Fig. 6b, the tangential force equals the friction force; therefore, the filament slides on the surface to Fig. 6c. In Fig. 6c, it is assumed that the filament sticks to the surface (i.e. it impacts a barrier, e.g. a biofilm agglomerate). The fixed point is indicated by a dot in the diagrams. In Fig. 6d, the filament undergoes a further bending until it reaches the last position before flipping (Fig. 6e). The filament will then flip; Fig. 6f shows the flipped shape. Moving further over the flip point will accordingly result in Fig. $6 \mathrm{~g}$ and Fig. 6h. In the position shown in Fig. 6h, the normal force becomes negative; thus, the filament is drawn out of the sticking site and then slides along the tooth surface with a relatively low force until the brush changes direction in Fig 6i.

The described motion of the filament is one of many possible motions. These motions depend on many details, such as the local friction coefficient that may vary due to saliva, toothpaste particles and biofilms. We wish to describe these influences in further detail by discussing Fig. 6 in the context of the transversal forces shown in Fig. 7.

In Fig. 7, the transversal force in $x^{\prime}$-direction is shown versus the relative tip to bearing distance $w_{t i p}$ of the filament. We have chosen the negative sign in $w_{\text {tip }}$ to explain the filament shape from left to right, i.e. in the same direction as in Fig. 6. The red dots demonstrate the transversal forces of the filament shapes shown in Fig. 6. 

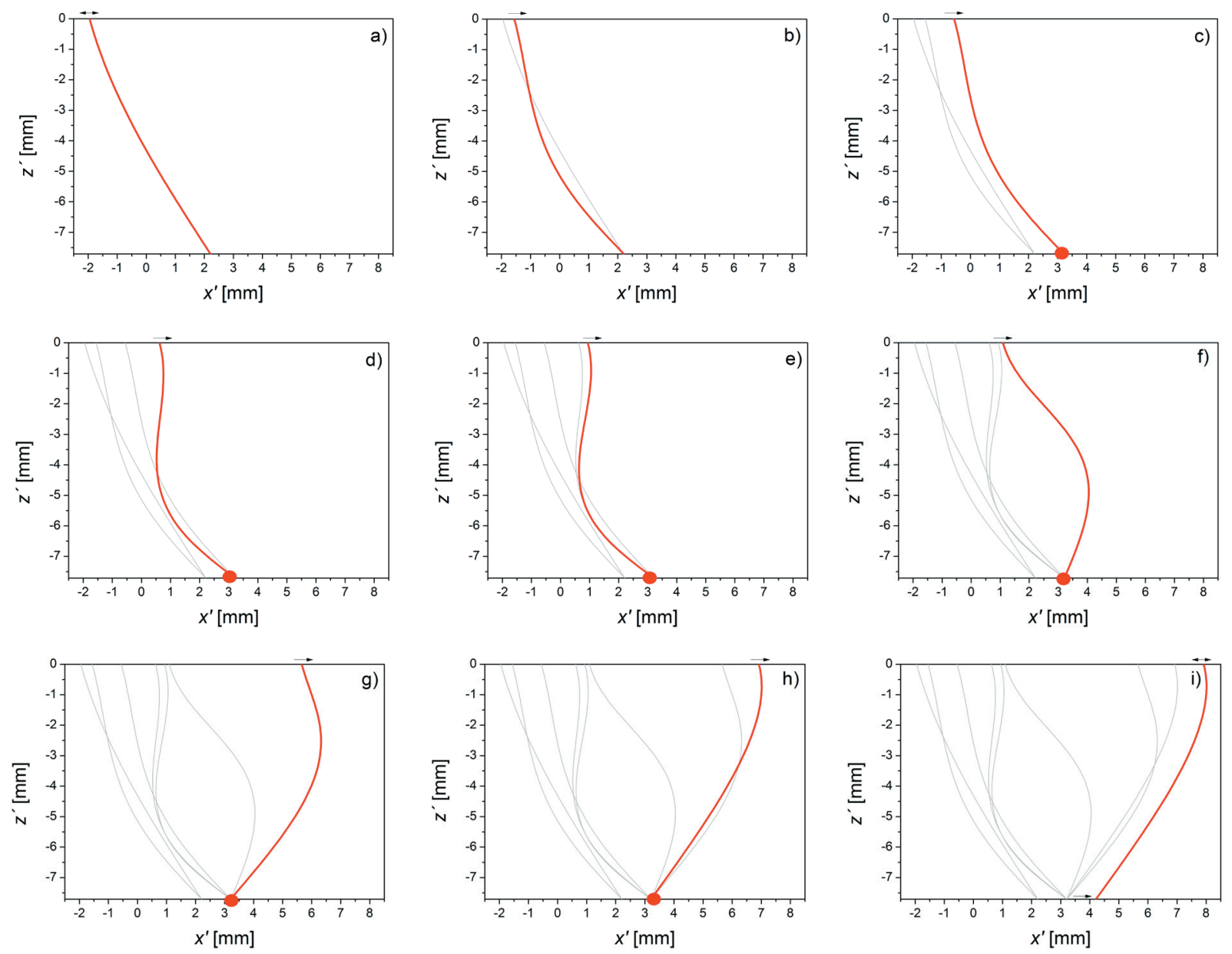

Fig. 6. Bending line of a simplified toothbrush filament with $d_{0}-d=0.75 \mathrm{~mm}$ for different brush head positions

The full curves show sliding/sticking solutions. The filament may slide along the tooth surface with a constant shape as long as the friction force equals the transversal force of the filament. The dashed lines refer to sticking solutions.

Here, the slope of the transversal force versus tip position is negative and, according to Eq. (9), the filament shape is unstable. Physically speaking, this means that the filament tip sticks to the surface while the bearing moves; therefore, the shape varies permanently. The first filament shape (Fig. 6a) shows only a very low positive transversal force. This force increases (here in the negative direction, i.e. against the movement of the filament) until the transverse force and the friction force are equal at the second filament shape. This shape then remains constant, and the tip slides along the tooth surface until the friction force exceeds the maximum possible transverse force. In Fig. 6, it is assumed that this sliding condition is valid for $1 \mathrm{~mm}$; therefore, the filament shapes in Figs.
$6 \mathrm{~b}$ and $\mathrm{c}$ are equal but separated by $1 \mathrm{~mm}$ of brush movement. We further assume that the filament suddenly becomes stuck, and the sticking force exceeds the maximum transverse force that is reached in Shape 3. If the brush moves further, the filament sticks to the tooth and flips between Shapes 4 and 5 in Figs. 6e and f. Next, the transversal force changes direction, i.e. it points against the direction of the brush movement. Depending on the friction force, in the backward direction it is possible that the filament's tip moves backwards after that point, but for the sake of clear graphic representation it is assumed that the filament remains stuck even at Shape 6 with the highest backward force. The transversal force then decreases rapidly until the transversal force again becomes negative (Shape 7). Here, the transversal force is relatively small; nevertheless the friction force is overcome. This is because the normal force (shown in Section 2.1.2) becomes negative, i.e. the filament is pulled upwards out of the sticking site. Consequently, 
the filament slides over the surface until the direction of movement is changed.

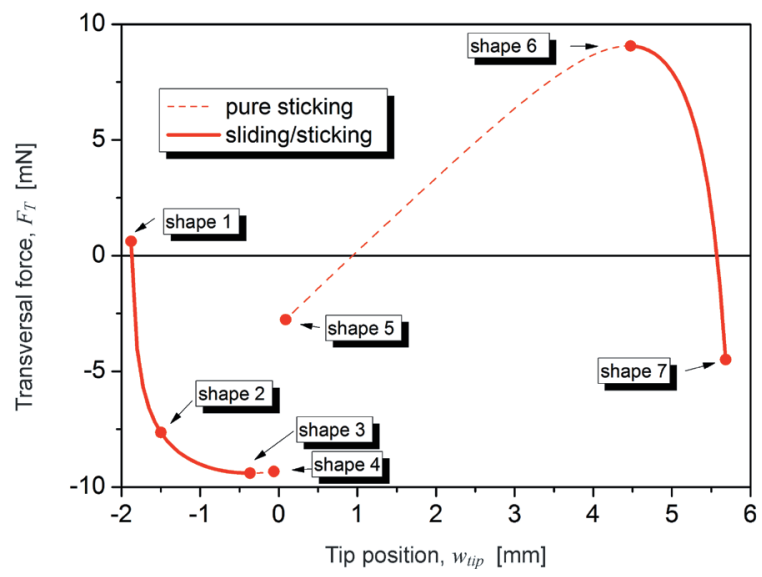

Fig. 7. Bending line at different tip positions for a filament with $\alpha=16^{\circ}$ and $d_{0}-d=0.75 \mathrm{~mm}$

\subsubsection{Solution: Forces}

As seen in Section 2.1.1, the transversal force $F_{t}$ and the normal force $F_{n}$ play an important role for the modelling of the filament motion. These forces are shown in Figs. 8 and 9 for inclination angles of $\alpha=16^{\circ}$ and $\alpha=0^{\circ}$ at a distance of $d_{0}-d=0.75 \mathrm{~mm}$.

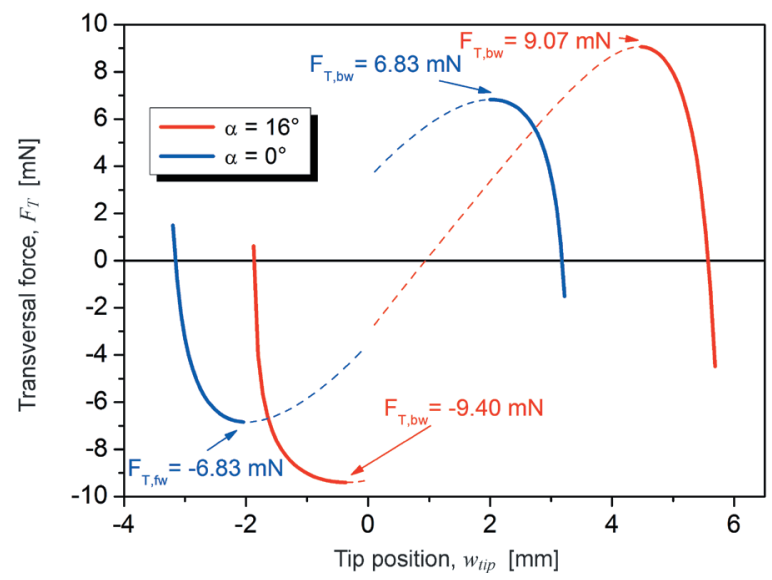

Fig. 8. Transversal force $F_{T}$ versus tip position $w_{\text {tip }}$ $\left(\alpha=16^{\circ}\right.$ and the $\alpha=0^{\circ} d_{0}-d=0.75 \mathrm{~mm}$ )

The transversal force (Fig. 8) obviously takes its maximum value at the border between the sliding/ sticking and the sticking solutions. The maximum transversal force for the inclined filament is significantly higher than for the vertical one.

The two curves for the normal force in Fig. 9 show similar behaviour. The maximum normal forces $F_{N}$ for both $\alpha=16^{\circ}$ and $\alpha=0^{\circ}$ are defined as the normal forces at the border between the sliding/ sticking and the sticking solutions, i.e. at the position of maximum transversal force. They are the maximum values in the sliding/sticking range, but even higher values are reached in the pure sticking range. Again, the maximum normal forces for the $16^{\circ}$-inclined filament are higher (only slightly for the forward direction) compared to the vertical filament.

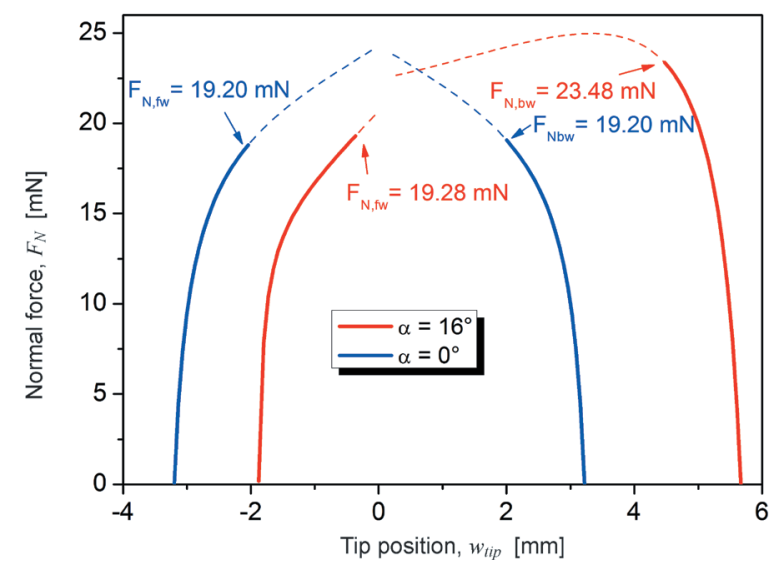

Fig. 9. Normal force $F_{N}$ versus tip position $w_{\text {tip }}$ ( $\alpha=16^{\circ}$ and $\alpha=0^{\circ} d_{0}-d=0.75 \mathrm{~mm}$ )

These curves have been analysed for all geometries with the four defined brush-to-tooth distances.

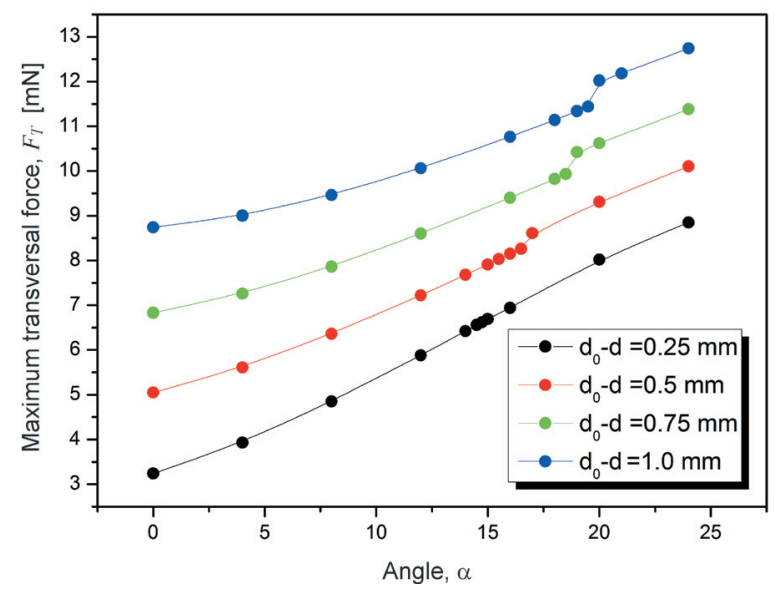

Fig. 10. Maximum transversal force $F_{T}$ in the sliding/sticking branch (shape 3 in Fig. 7) versus inclination angle

Fig. 10 shows the maximum transversal force in the sliding/sticking region over the filament angle $\alpha$ for different filament compressions $d_{0}-d$. The results show that the transversal force increases with increasing inclination angle independent of the filament compression $d_{0}-d$. For a typical $d_{0}-d$ of 0.5 
$\mathrm{mm}$, about $60 \%$ more transversal force is obtained for a $16^{\circ}$ angled filament compared to a vertical one.

In Fig. 11, the maximum normal forces to the surface are plotted over the filament angle for different $d_{0}-d$ values. The results show that for $d_{0}-d \leq 0.75$ $\mathrm{mm}$ the maximum normal forces increase slightly with the filament angle up to the angle of about $16^{\circ}$; for the very small value of $d_{0}-d=0.25 \mathrm{~mm}$, the increase ends at an angle of $\alpha=14.75^{\circ}$. Above this value, the maximum normal forces strongly decrease. For $d_{0}-d=1 \mathrm{~mm}$ the maximum normal forces slightly decrease (about 3\%) with the angle but strongly decrease for angles $>19^{\circ}$.

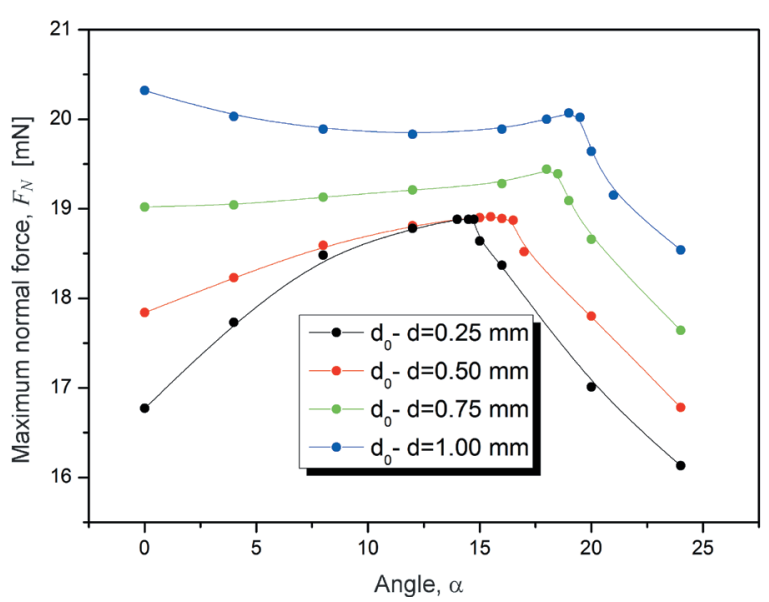

Fig. 11. Maximum normal force $F_{N}$ in the sliding/sticking branch (shape 3) versus $\alpha$

This steep decrease of the normal force is the reason the angle of $16^{\circ}$ is considered to be optimal for cleaning purposes. Details on this argumentation are given in the discussion section.

\subsection{Penetration Model}

Fig. 12 shows the results for the penetration model for a $16^{\circ}$ filament and a brush deformation of $d_{0}-d=1 \mathrm{~mm}$.

All filaments are shown in their most backward position, i.e. equivalent to Shape 7 in the tooth surface model (Fig. 7).

The filament is moved from right to left in a forward direction and touches the tooth flank as a straight filament (Filament 1). When moving further, the filament penetrates deeper into the flank (Filament 2) until it reaches its deepest penetration (Filament 3); the related angle for our tooth model is $\varphi_{16^{\circ}}=69^{\circ}$. After that turning point, the filament moves upwards again (Filaments 4 and 5) until it reaches the flat tooth surface (Filament 6).

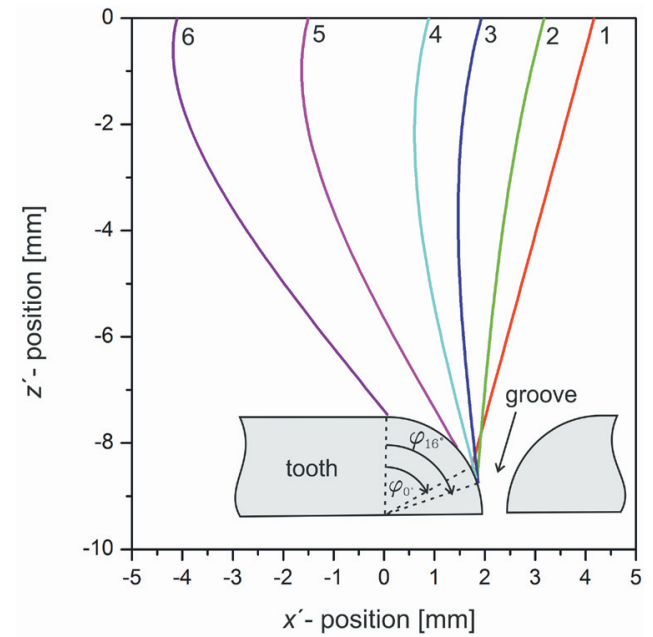

Fig. 12. Filament shapes depending on the brush position

$$
\left(\alpha=16^{\circ}, d_{0}-d=1 \mathrm{~mm}\right)
$$

In comparison, the filament with a zero inclination angle would touch the tooth flank at the same position as the $16^{\circ}$-filament but would not be able to penetrate deeper into the flank. Therefore, the maximum reachable angle is $\varphi_{0^{\circ}}=60^{\circ}$. Consequently, the $16^{\circ}$-filament penetrates the gap deeper by $9^{\circ}$ or $0.31 \mathrm{~mm}$, which is equivalent to a $15 \%$ increase in the penetrated area.

A brush movement of approximately $8 \mathrm{~mm}$ is necessary to reach all positions from the red to the purple curve. Therefore, a filament from the oscillating brush would only reach a portion of these positions depending on its starting position. Nevertheless, only about a 2-mm travel range is necessary to reach the deepest penetration in Curve 3.

\section{NUMERICAL SIMULATIONS}

The problem of filament shapes on the flat tooth surface model was numerically solved in order to judge whether the simplifications of our analytical model lead to valid results. The finite element method (FEM) with the COMSOL Multiphysics commercial software package (COMSOL, Inc. Burlington, USA) was used. A 2D model of the beam with equivalent second moment of inertia was implemented. The displacement was introduced on the neutral axis at the beam end while the other end was kept fixed. Since large deformations have to be included, geometric nonlinearities were considered in the model. The problem was solved on a triangular mesh with approximately 700 elements. In the FEM model, the same boundary conditions were used as in our analytical model, but include the effect of 
high displacements that is neglected in the analytical theory.

The resulting bending lines for the $16^{\circ}$-filament and $d_{0}-d=0.75 \mathrm{~mm}$ are shown in Fig. 13. It is clearly shown that both methods agree very well for negative tip displacements, i.e. for Shapes 1 to 3, but our analytical model loses accuracy in the range of high positive tip displacements, i.e. for Shapes 6 and 7.

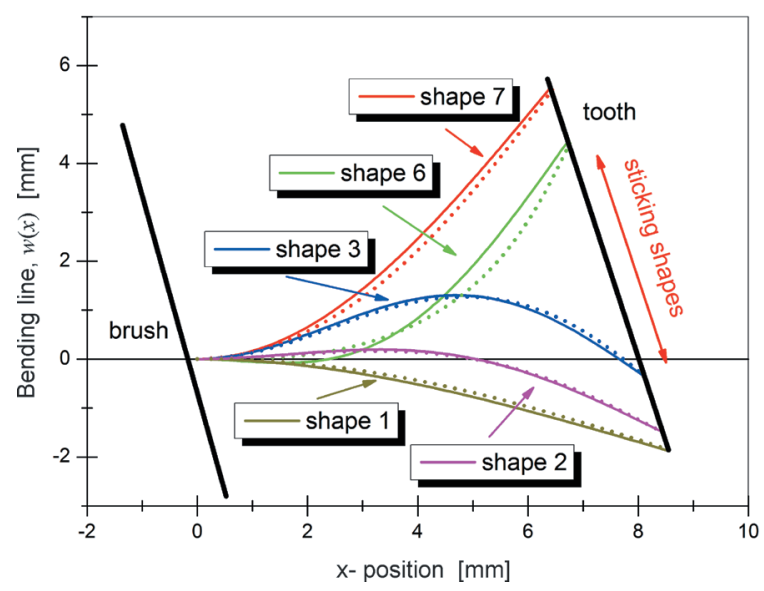

Fig. 13. Filament bending lines $\left(\alpha=16^{\circ}, d-d_{0}=0.75 \mathrm{~mm}\right)$ proposed model (full lines) and FEM simulations (dotted lines)

The same is true for the derived normal and transversal forces shown in Figs. 14 and 15. In the worst case, near the point of maximum force, the calculated (analytical) maximum normal and transversal forces are approximately a factor of two higher than the simulated (FEM) ones. This is supposed to be caused by the assumption of a spatially constant axial force in the analytical theory. In reality, the axial force is reduced due to a spatially varying bending away from the axial direction. Consequently, the analytical theory overestimates the normal force. The transversal force must therefore also be overestimated to compensate this axial force overestimation and to reach the same displacement as in the simulation. This argument also holds for the range of very high displacements (near Shape 7). As the normal force is reduced to zero, its effect on the filament shape vanishes and the deviation from the simulation is reduced. As a result, it must be stated that, for filaments with large strokes and large normal forces, i.e. for filaments in the range of Shape 6 , the forces are significantly overestimated by the analytical theory. Nevertheless the analytical theory leads to a fairly good agreement for small displacements (Shapes 1 to 3) and for low normal forces (Shape 7).
A manual brush will reach all shapes, from 1 to 7 , due to its high stroke. For these brushes, the analytical theory overestimates the cleaning effect, but the general trend derived from the analytical theory remains valid. Fortunately, the filaments of the oscillating-rotating brush will predominantly be bending in Shapes 1 to 3, where the analytical model coincides well with simulated curve. Therefore, the model quantitatively suits well the main goal of this paper, which is the characterization of an oscillatingrotating brush with $16^{\circ}$-inclined filaments.

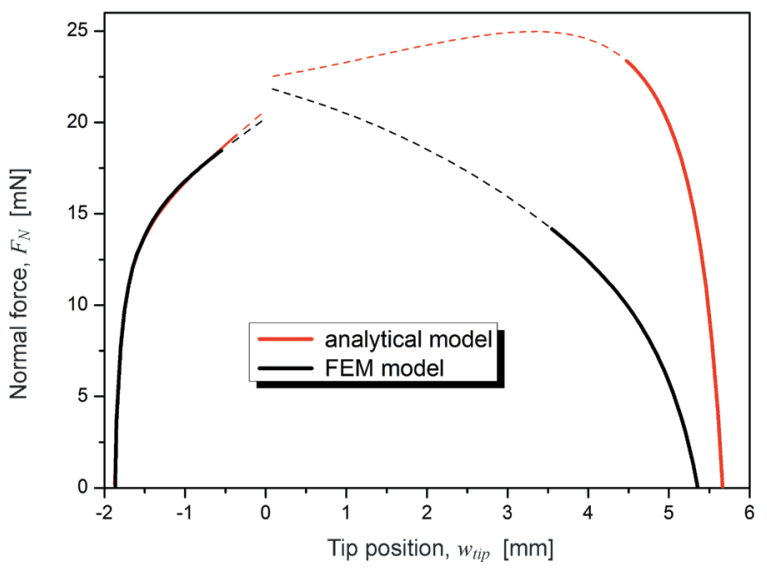

Fig. 14. Normal forces versus tip position $\left(\alpha=16^{\circ}, d_{0}-d=0.75 \mathrm{~mm}\right)$

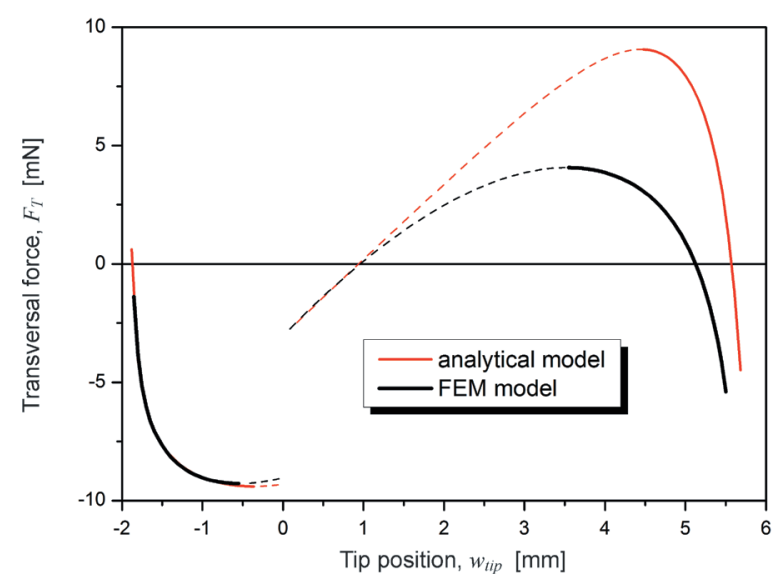

Fig. 15. Transversal forces versus tip position $\left(\alpha=16^{\circ} d_{0}-d=0.75 \mathrm{~mm}\right)$

\section{EXPERIMENTAL VALIDATION}

A rheometer (Thermo Fisher Scientific, HAAKE Rheostress1) was used to qualitatively validate the increasing shear force with $16^{\circ}$-inclined filaments. With the rheometer, the transferred torque from the brush head to a simplified tooth surface could be 
measured (according to our simplified surface model). The transferred momentum is proportional to the transversal (shear) force, since the two brush heads have the same tufting configuration. The spread of the angled filament brush head that slightly increases the radius of the outside filament arrangement (outer ring) compared to the straight filament brush head can be ignored.

In the experiment, a brush head with the straight filaments and a brush head with the $16^{\circ}$-inclined filaments were compared (Fig. 2). The unidirectional rotational speed of the brush head and the normal force that is applied to the brush head were controlled, and the transferred torque to a sandblasted flat plastic plate was measured (typically used in in-vitro brushing tests). The rotation speed was controlled at $1 \mathrm{rpm}$, and its direction was changed (i.e. oscillated) after one full rotation. The normal force was controlled using weights on a balance (50 to $390 \mathrm{~g}$ ) in such a way that the plastic plate was pressed with constant force against the oscillating-rotating brush head.

The transferred torque data were averaged and are given in Fig. 16.

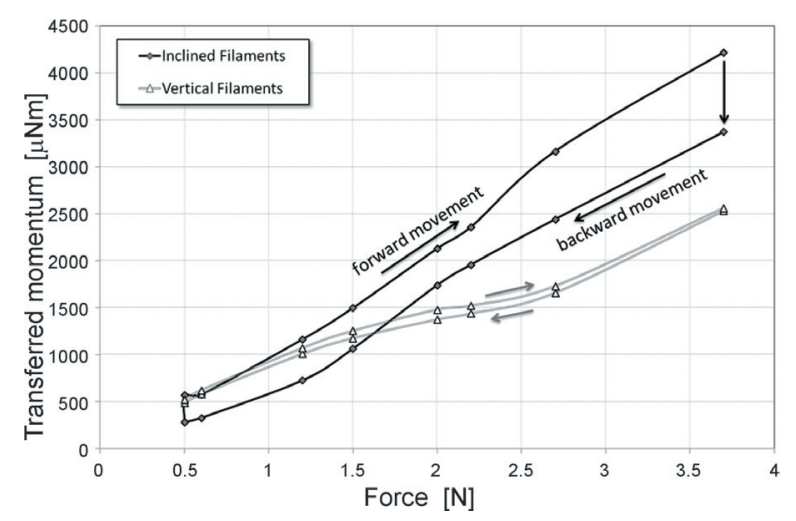

Fig. 16. Transferred force versus normal force for two brushes with $\alpha=0^{\circ}$ and $\alpha=16^{\circ}$

For small normal forces, both brush heads showed the same transferred torque. With increasing normal force, the forward movement (rotation) of the brush head with $16^{\circ}$ angled filaments transferred significantly higher torques than the brush head with straight filaments. Oral-B's advanced electric toothbrushes feature a pressure sensor that guides the user not to apply too much brushing pressure. This pressure sensor typically lights up at pressure/brushing forces above $2.5 \mathrm{~N}$. At this recommended maximum brushing force, the brush head with $16^{\circ}$ filaments was able to transfer about a factor of 1.8 more torque to the test plate. The experiment was repeated on other materials (steel, ceramics) and similar qualitative behaviour as in Fig. 16 was found.

\section{DISCUSSION}

An analytical model for the bending of $16^{\circ}$-inclined toothbrush filaments under the combined action of normal and transversal forces was developed and compared to numerical FEM solutions. It turned out that our analytical model was valid for small filament displacements, but became inaccurate with high filament displacements. As a consequence, for the given geometry of the oscillating-rotating brush, our model can be used for the defined Shapes 1 to 3 (Fig. 7), and to a lesser extent Shape 7, but overestimates the forces for the other sliding positions near Shape 6 .

Our model was used to calculate the forces that act on a tooth depending on the brush to tooth distance $d$. In this section, how these observations for single filaments on idealized tooth geometries are related to the behaviour of a full brush on a real tooth are discussed. The basic idea is that a brush consists of many filaments that touch the tooth under different conditions. They may be bent differently due to different friction coefficients at different locations on the tooth and at different distances $d$ due to the uneven tooth structure. Furthermore, the brush consists of different filaments made from different materials and cut to different lengths. A prediction of full brush behaviour from the collective action of the filaments requires significant simplification.

It is supposed that at any given time some filaments work in the most favourable way for cleaning while others are in a condition that does not contribute significantly to the cleaning process. Optimizing the best possible conditions of a filament, therefore, optimizes the overall cleaning success. It is supposed that the transversal (shear) force is the most important parameter in teeth cleaning, but also that a certain amount of normal force is necessary. Therefore, the transversal force should be maximized by choosing the optimum angle $\alpha$ such that the normal force is not reduced compared to the zero angle case.

One important factor for this discussion is the stroke length of the brush. The manual brush may be used with a stroke of $10 \mathrm{~mm}$ or more [3]. Consequently, all possible shapes from Fig. 6 may be accessed. The manual brush using $16^{\circ}$-angled filaments has already proved to have significantly greater plaque removal compared to straight brushes in experimental trials [3]. This result may be explained by the fact that the transverse forces for the $16^{\circ}$-angled filament are higher compared to the straight filament 
over the entire range of possible shapes (Fig. 7). However, the results for high filament displacements are overestimated by the model, as shown earlier (Section 3: Numerical simulations).

When considering the oscillating-rotating brush, we must account for the reduced strokes compared to the manual brush. Here the stroke is reduced to 2.3 $\mathrm{mm}$ (inner ring) and to $4.0 \mathrm{~mm}$ (outer ring). Therefore, the shapes from Figs. 6a to e are relevant, while the other shapes play a lesser role. Fortunately, the model proved to be accurate for these shapes (Shapes 1, 2 and 3). As a result, we conclude that the model is appropriate to describe the cleaning process for the oscillating-rotating brush, despite its inaccuracy for high filament displacements. The calculated maximum normal and transversal forces in the range between Shapes 1 and 3 versus the inclination angle for the four calculated distances are shown in Figs. 10 and 11. It is clearly shown that the transversal force grows with the inclination angles for all distances, while the normal force shows a more complex behaviour. Depending on the distance $d$, the normal force may rise or fall slightly with the inclination angles for low angles, but all curves start to decrease with a steep slope for angles higher than about $16^{\circ}$. Therefore, the optimum angle is supposed to be $16^{\circ}$. At this angle, the transversal force is increased by $60 \%$ compared to the common vertical configuration.

A second important parameter for the cleaning performance of a toothbrush is the penetration depth into approximal areas. Again, the full behaviour of the brush cannot be predicted, but we assume that some filaments will penetrate to the deepest amount possible into a given gap. Therefore, optimizing the maximum possible penetration depth is a good measure for the overall penetration performance. As the $16^{\circ}$-inclined filaments are longer than the vertical ones for the same brush thickness $d_{0}$, it is likely that they may penetrate deeper into a gap, which was demonstrated for the $16^{\circ}$-filament. For the defined tooth geometry, the filament was able to penetrate about $0.3 \mathrm{~mm}$ deeper into the cavity. This corresponds to a $15 \%$ increase of the cleaned area; in experimental studies [3], the inclined filaments of the manual CrossAction ${ }^{\circledR}$ brush performed $10 \%$ better on penetration per stroke compared to commercially available brushes.

This study was conducted to improve the theoretical understanding of the filament-tooth interaction. Experimental studies typically consider the total normal brushing force and its impact on measures, such as efficacy and gingival abrasion [13] or dentin and enamel abrasion [14]. This study, however, shows how the physical process of global brush deformation $\mathrm{d}_{0}$-d, which is closely related to the global normal force, leads to a variety of normal and transverse forces acting on the tooth surface or biofilm, respectively, depending on the individual filament position. Thus, it details the causal chain from global normal force to the mechanical impact on dental plaque as a prerequisite for the understanding of efficient removal. This approach is intended to help understand the physical impact of toothbrush filaments on tooth surfaces, which have been described as causative factors of cleaning efficacy or soft and hard tissue abrasion. Ultimately, such considerations will help to draw implications for a better toothbrush design. As with any consumer product of this kind, the final proof of efficacy benefit needs to be verified under in-use conditions, such as a consumer or clinical study.

\subsection{Implication for Toothbrush Design}

Based on this study, some design conclusions for brush heads can be drawn:

1) Optimize filament action: To achieve the maximum transversal (shear) force during a brushing stroke on a flat surface, the filament has to reach Shape 3 in Fig. 7. This corresponds to a calculated stroke length of $2.32 \mathrm{~mm}\left(d_{0}-d<1 \mathrm{~mm}\right)$ for a straight filament and $0.53 \mathrm{~mm}\left(d_{0}-d<1 \mathrm{~mm}\right)$ for a $16^{\circ}$-inclined filament plus the distance of sliding prior to the stuck event (Shape 3 in Fig. 7).

2) Arrange filaments with angle: In order to increase the maximum absolute shear force and the penetration into approximal areas, the filament should be angled towards the surface.

3) Optimize the filament angle: With an increasing filament angle up to about $16^{\circ}$, the shear force increases while the normal force remains approximately constant. However, the filament angle should not be larger than about $16^{\circ}$, because the normal force decreases above this value and becomes smaller than the vertical filament version, which we wish to avoid.

4) Deliver maximum number of efficacy events: The brushing efficacy increases with the number of maximum shear force events per time interval. The maximum frequency is the frequency in which filaments can still travel by at least a stroke length of $0.53 \mathrm{~mm}$.

5) Ensuring travel of filaments at tooth surface: The configuration of the brush head (filament diameter/length, tuft arrangements, etc.) has to be designed in such a way that the movement of the brush head can be transferred across the filaments 
towards the tooth surface, i.e. it must be ensured that the movement is not reduced/suppressed with increasing brushing force.

\section{CONCLUSIONS}

According to the derived model, the simulation data, and our experimental validation, we conclude that we can further increase the efficacy of a toothbrush in terms of biofilm removal. We have shown that oscillating-rotating electric toothbrushes deliver the necessary filament displacement (travel) to exert maximum shear force to the biofilm.

These high efficacy events occur during the deformation of the filament, just before filament flipping. In comparison to manual brushes, the electric toothbrush can increase the number of these high efficacy events during brushing (e.g. 78 events per second for a typical $\mathrm{O} / \mathrm{R}$ power brush).

We have clearly demonstrated that filaments that are inclined result in higher shear forces, which is beneficial for plaque removal. A filament angle of about $16^{\circ}$ was found to maximize shear force, while keeping the normal force almost constant compared to similar brush heads with straight filaments. Furthermore, the penetration into approximal areas can be clearly increased using $16^{\circ}$-inclined filaments, in comparison to straight filaments.

A filament angle of $16^{\circ}$ has proved to increase plaque removal in the design of manual brushes. Our study shows that this angle also applies for oscillating-rotating power brushes. The new Oral-B CrossAction ${ }^{\circledR}$ replacement brush head features an outer and an inner ring with inclined filaments. The outer ring has an angle of $+16^{\circ}$ for the forward direction of the oscillation; the inner ring has an angle of $-16^{\circ}$ for the backward direction of the oscillation. Both the outer and the inner ring have sufficient deflection to exert maximum shear forces. The inner part of the brush head shows only minor movement. This part was designed with straight filaments to provide the necessary stability (stiffness) of the filament design. The brush head in Fig. 2 was designed to meet the above-derived criteria for optimized shear force transmission and increased approximal area penetration (for the given oscillation frequency) and provides sufficient filament tip motion if used with the recommended brushing force (maximum $2 \mathrm{~N}$ ). Based on our knowledge of the forces needed for biofilm removal, the Oral-B CrossAction ${ }^{\circledR}$ brush head with filament angles of $16^{\circ}$ can deliver superior plaque removal efficacy in comparison to other tuft designs. These benefits have been demonstrated in clinical investigations, which are currently in the publication process.

\section{ACKNOWLEDGEMENT}

The authors wish to thank Oral-B Laboratories for their financial support of this study.

\section{REFERENCES}

[1] Do, T., Devine, D., Marsh, P.D. (2013) Oral biofilms: molecular analysis, challenges, and future prospects in dental diagnostics. Clinical, Cosmetic and Investigational Dentistry, vol. 5, p. 11-19, DOI:10.2147/CCIDE.S31005.

[2] Teughels, W., Van Assche, N., Sliepen, I., Quirynen, M. (2006). Effect of material characteristics and/or surface topography on biofilm development. Clinical Oral Implants Research, vol. 17, suppl. 2, p. 68-81, DOI:10.1111/j.1600-0501.2006.01353.x.

[3] Beals, D., Ngo, T., Feng, Y., Cook, D., Grau, D.G., Weber, D.A. (2000). Development and laboratory evaluation of a novel brush head design. American Journal of Dentistry, vol. 13, special no., p. 5A-14A.

[4] Cugini, M.A., Warren, P.R. (2006). The Oral-B ${ }^{\circledR}$ CrossAction ${ }^{\circledR}$ manual toothbrush: A 5-year literature review. Journal of the Canadian Dental Association, vol. 72 , no. 4 , p. 323a-k.

[5] Cugini, M., Thompson, M., Warren, P. R. (2006). Correlations between two plaque indices in assessment of toothbrush effectiveness. The Journal of Contemporary Dental Practice, vol. 7, no. 5, p. 1-9.

[6] Terézhalmy, G.T., Biesbrock, A.R., Walters, P.A., Grender, J.M., Bartizek, R.D. (2008). Clinical evaluation of brushing time and plaque removal potential of two manual toothbrushes. International Journal of Dental Hygiene, vol. 6, no. 4, p. 321-327, DOI:10.1111/j.1601-5037.2008.00327.x.

[7] Sharma, N.C., Qaqish, J., Walters, P.A., Grender, J., Biesbrock, A.R. (2010). A clinical evaluation of the plaque removal efficacy of five manual toothbrushes. Journal of Clinical Dentistry, vol. 21, no. 1, p. 8-12.

[8] Sharma, N.C., Qaqish, J.G., Galustians, H.J., King, D.W., Low, M.A., Jacobs, D.M., Weber, D.A. (2000). A 3-month comparative investigation of the safety and efficacy of a new toothbrush: results from two independent clinical studies. American Journal of Dentistry, vol. 13, special no., p. 27A-32A.

[9] Robinson, P.G., Deacon, S.A., Deery, C., Heanue, M., Walmsley, A.D., Worthington, H.V., Glenny, A.M., Shaw, W.C. (2005). Manual versus powered toothbrushing for oral health. Cochrane Database of Systematic Reviews, vol. 2, p. CD002281, DOI:10.1002/14651858.CD002281.pub2.

[10] Yacoob, M., Deacon, S.A., Deery, C., Glenny, M., Walmsley, A.D., Worthington, H., Robinson, P.G. (2011). Manual vs. powered toothbrushes for oral 
health: Updated Cochrane Review. British Society for Oral and Dental Research Meeting Presentation.

[11] Budak, I., Trifkovic, B., Puskar, T., Vukelic, D., Vucaj-Cirilovic, V., Hodolic, J., Todorovic, A. (2013). Comparative analysis of $3 \mathrm{D}$ digitization systems in the field of dental prosthetics. Technical Gazette, vol. 20, no. 2, p. 291-296.

[12] Popov, E.P. (1998). Engineering Mechanics of Solids, Second Edition. Prentice Hall, Upper Saddle River.
[13] Van der Weijden, G.A., Timmerman M.F., Versteeg, P., Piscaer, M., Van der Velden, U. (2004). High and low brushing force in relation to efficacy and gingival abrasion. Journal of Clinical Periodontolgy, vol. 31, no. 8, p. 620-624, DOI:10.1111/j.1600-051x.2004.00529.x

[14] Wiegand, A., Burkhard, J.P.M., Eggmann, F., Attin, T. (2013). Brushing force of manual and sonic toothbrushes affects dental hard tissue abrasion, Clinical Oral Investigations, vol 17, no. 3, p. 815-822, DOI:10.1007/s00784-012-0788-z. 